九州大学学術情報リポジトリ

Kyushu University Institutional Repository

\title{
A NOTE ON DISCRETE MARKOVIAN DECISION PROCESS
}

Ogawara, Masami

Tokyo Woman's Christian College

https://doi.org/10.5109/13012

出版情報：統計数理研究. 11 (1/2)，pp. 35-42，1964-03. Research Association of Statistical Sciences

バージョン :

権利関係 : 


\title{
A NOTE ON DISCRETE MARKOVIAN DECISION PROCESSES
}

\author{
By \\ Masami OGaWARA
}

(Received November 15, 1963)

Introduction. In the current method of policy improvement for Markovian decision processes, it seems to be assumed that the optimal policy is composed of successions of the same decision. (Howard [2], Blackwell [3]) When the discount factor $\beta$ is less than one, this fact is easily proved by use of Blackwell's theorems ( $\$ 2)$. In the case of $\beta=1$, as we shall show in $\$ 3$, similar theorems hold but the situation is not affirmative. In the last paragraph (\$4) we shall do some generalizations of discrete Markovian decision processes.

$\S 1$. Definitions and Notations. In the following, symbol $\equiv$ means definition or identity. We consider a system such that the states of which are labeled by the integers and form a finite set $S \equiv\{1,2, \ldots, N\}$. We assume that the set $A$ of our possible actions with the system is also finite; $A \equiv\{1,2, \cdots, \mathrm{M}\}$. In this note however these assumptions of finiteness are not essential.

Decision function $d=d(i)$ is a mapping from i $\in S$ onto $d \in A$, and the set $D$ of decision functions is finite; the number of its elements is $M^{N}$.

Now, we observe the system at intervals of unit time and make a decision on our action according to the state. In that case we assume that the system moves with the transition probability matrix

$$
P(d) \equiv\left(p_{i j}(d)\right)
$$

which depends on the decision function $d$ and that we receive an immediate reward $r_{i j}(a)$ for one-step transition $i \rightarrow j$ and an action $a \in A$, where $r_{i j}(a)$ is a random variable. The expected immediate reward is then

$$
r(i, d(i)) \equiv \sum_{j} p_{i j}(d(i)) r_{i j}(d(i))
$$

and

$$
r(d) \equiv\left(\begin{array}{c}
r(1, d(1)) \\
\vdots \\
r(N, d(N))
\end{array} \quad(d \in D)\right.
$$

is said to be an expected immediate reward vector. 
By a policy $\pi$, we mean a sequence of decision functions ;

$$
\pi \equiv\left(d_{1} d_{2} d_{3} \cdots\right) \quad\left(d_{n} \in D, n=1,2, \cdots\right)
$$

According to Blackwell, we shall use the following notations for various special policies; for a policy $\pi \equiv\left(d_{1} d_{2} \cdots\right)$

$$
\begin{aligned}
& (d \pi) \equiv\left(d d_{1} d_{2} \cdots\right) \\
& \left(g_{1} \cdots g_{n} \pi\right) \equiv\left(g_{1} \cdots g_{n} d_{1} d_{2} \cdots\right) \\
& \left(d^{(n)} \pi\right) \equiv(\underbrace{d d \cdots d}_{n} d_{1} d_{2} \cdots) \\
& d^{(\infty)} \equiv(d d \cdots) \\
& (T \pi) \equiv\left(d_{2} d_{3} \cdots\right)
\end{aligned}
$$

and so forth. We denote the set of all policies by $\Pi$.

The $n$-step transition probability matrix for a policy $\pi \equiv\left(d_{1} d_{2} \cdots\right)$ is denoted by

where

$$
P_{n}(\pi) \equiv P\left(d_{1}\right) P\left(d_{2}\right) \cdots P\left(d_{n}\right) \quad(n=0,1,2, \cdots),
$$

Finally, if the present value of unit income $n$-steps in future is $\beta^{n}$, the $\beta$ is called a discount factor; if the unit time interest rate is $\rho, \beta=(1+$ $\rho)^{-1}$ and $0<\beta \leqq 1$.

§ 2. Optimal Policies for $\beta<1$. If $\beta<1$ and $r(d)$ has finite components on $D$, the expected total rewards vector corresponding to a policy $\pi \equiv$ $\left(d_{1} d_{2} \cdots\right)$ is given by the convergent infinite series

$$
\begin{aligned}
V(\pi) & =\sum_{n=0}^{\infty} \beta^{n} P_{n}(\pi) r\left(d_{n+1}\right) \\
& =r\left(d_{1}\right)+\beta P\left(d_{1}\right) V(T \pi) ;
\end{aligned}
$$

the ith component of $N \times 1$ column vector $\boldsymbol{V}(\pi)$ is the expectation of total rewards when the process started from a state $i$.

Now we define a semi-order in the $N$ dimensional vector space $\mathfrak{v}$. For two $N \times 1$ column vectors

$$
v_{1}=\left(\begin{array}{l}
v_{11} \\
\vdots \\
v_{1 N}
\end{array}\right) \quad, \quad v_{2}=\left(\begin{array}{c}
v_{21} \\
\vdots \\
v_{2 N}
\end{array}\right)
$$

if $v_{1 i} \geqq v_{2 i}(i=1,2, \cdots, N)$ we denote $v_{1} \geqq v_{2}$ or $v_{2} \leqq v_{1}$

and if $v_{1} \geqq v_{2}$ and $v_{1} \neq v_{2}$ we denote $v_{1}>v_{2}$ or $v_{2}<v_{1}$.

The join of two vectors $v_{1}$ and $v_{2}, v_{1} \vee v_{2}$, is defined by the vector whose $i$ th component is $\max \left(v_{1 i}, v_{2 i}\right)$ and the meet $v_{1} \wedge v_{2}$ is the vector whose $i$ th 
component is $\min \left(v_{1 i}, v_{2_{i}}\right)$. Thus $\mathfrak{v}$ is a vector lattice and the absolute value of $v(v \in \mathfrak{b})$ is defined as a vector whose ith component is given by $v_{i}$. If we define, morerover, the norm of $v$ by $v \equiv \max _{1 \leq i \leq . .} v_{i}, \mathfrak{b}$ is a Banach lattice. The subspace of $\mathfrak{v}, \mathfrak{p}(I)=\{V(\pi) ; \pi \in \Pi\}$, which is induced by (2.1) from $I$, is also a Banach lattice.

Corresponding to space $\mathfrak{b}$, we introduce the semi-order into the policy space $\Pi$; for two policies $\pi_{1}$ and $\pi_{2}$, we write

$$
\begin{array}{lll} 
& \pi_{1} \geq \pi_{2} & \text { if } V\left(\pi_{1}\right) \geq V\left(\pi_{2}\right) \\
& \pi_{1}=\pi_{2} & \text { if } V\left(\pi_{1}\right)=V\left(\pi_{2}\right) \\
\text { and } & \pi_{1}>\pi_{2} & \text { if } V\left(\pi_{1}\right)>V\left(\pi_{2}\right) .
\end{array}
$$

The policy $\pi^{*}$ is said to be optimal if $\pi^{*} \geqq \pi$ for all $\pi \in \Pi$. If the subspace $\mathfrak{v}(\Pi)$ is complete, then such optimal policy $\pi^{*}$ exists.

If there exist policies $\pi^{\prime}, \pi^{\prime \prime}$ such that

$$
V\left(\pi^{\prime}\right)=V\left(\pi_{1}\right) \vee V\left(\pi_{2}\right), V\left(\pi^{\prime \prime}\right)=V\left(\pi_{1}\right) \wedge V\left(\pi_{2}\right)
$$

for any two policies $\pi_{1}, \pi_{2}$, then we may define

$$
\pi^{\prime}=\pi_{1} \bigvee \pi_{2}, \pi^{\prime \prime}=\pi_{1} \wedge \pi_{2} ;
$$

thus $\Pi$ would be a lattice and $V(I)$ a homomorphic mappimg from $I I$ into $\mathfrak{v}(I I)$.

In the following, the convergence of vector sequence may be understood in the sense of norm defined above.

Lemma 2.1. For any two policies $\pi \equiv\left(d_{1} d_{2} \cdots\right)$ and $\pi^{\prime} \equiv\left(d_{1}^{\prime} d_{2}^{\prime} \cdots\right)$

$$
\lim _{n \rightarrow \infty} V\left(d_{1}^{\prime} \cdots d_{n}^{\prime} \pi\right)=V\left(\pi^{\prime}\right)
$$

Proof. We have

$$
V\left(d_{1}^{\prime} \cdots d_{n}^{\prime} \pi\right)=\sum_{\nu=0}^{n-1} \beta^{\nu} P_{\nu}\left(\pi^{\prime}\right) r\left(d_{\nu+1}^{\prime}\right)+\beta^{n} P_{n}\left(\pi^{\prime}\right) \sum_{\nu=0}^{\infty} \beta^{\nu} P_{\nu}(\pi) r\left(d_{\nu+1}\right)
$$

and there exists a constant vector $R$ such that $\|r(d)\|<R<\infty(d \in D)$. Therefore, the norm of the second term on the right hand side is less than $\beta^{n} R /(1-\beta)$ and $\beta^{n} \rightarrow 0(n \rightarrow \infty)$. Thus the second term $\rightarrow 0(n \rightarrow \infty)$.

Following to Blackwell we define 'monotone' operator $L(d)$ by

$$
L(d) v \equiv r(d)+\beta P(d) v(v \in \mathfrak{v}, \mathrm{d} \in D) .
$$

Then, if $v_{1} \geqq v_{2}$ or $v_{1}>v_{2}, L(d) v_{1} \geqq L(d) v_{2}$. The following two theorems are easily proved by lemma 2.1 and the monotoneity of operator $L(d)$. (Blackwell [3])

Theorem 2.1. If $\pi^{*}=\left(d \pi^{*}\right)$ for all $d \in D$, then $\pi^{*}$ is optimal. 
Theorem 2.2. If $(d \pi)>\pi$, then $d^{(\infty)}>\pi$.

Lemma 2.2. If $\pi_{1} \leqq \pi_{2}$, then $\left(d \pi_{1}\right) \leqq\left(d \pi_{2}\right)$ for all $d \in D$.

Proof. By the assumption $V\left(\pi_{1}\right) \leqq V\left(\pi_{2}\right)$. Hence $L(d) V\left(\pi_{1}\right) \leqq L(d)$ $V\left(\pi_{2}\right)$ or $V\left(d \pi_{1}\right) \leqq V\left(d \pi_{2}\right)$. Thus we get $\left(d \pi_{1}\right) \leqq\left(d \pi_{2}\right)$.

Theorem 2.3. If $\pi \equiv\left(d_{1} d_{2} \cdots\right)$ is optimal, then $\pi=d_{1}^{(\infty)}$.

Proof. Since $\pi \geqq\left(d_{2} d_{3} \cdots\right)$, we get by theorem 2.2

$$
d_{1}^{(\infty)} \geq\left(d_{2} d_{3} \cdots\right)
$$

and by lemma 2.2

$$
\left(d_{1} d_{1}^{(\infty)}\right) \geqq\left(d_{1} d_{2} \cdots\right) \text { or } d_{1}^{(\infty)} \geqq \pi,
$$

whereas $\pi$ is optimal, $\pi=d_{1}^{(\infty)}$.

Owing to this theorem we may search an optimal policy in the confined set of policies of the type $d^{(\infty)}(d=D)$ and the theorems 2.1 and 2.2 give the basis of policy improvement routine for $\beta<1$, by seting $\pi \equiv d_{1}^{(\infty)}\left(d_{1} \in D\right)$.

\section{§. Optimal Policies for $\beta=1$.}

The expected total reward for $n-1$ transitions under a policy $\pi \equiv\left(d_{1}\right.$ $\left.d_{2} \cdots\right)$ is given by

$$
V_{n}(\pi) \equiv \sum_{\nu=0}^{n-1} P_{\nu}(\pi) r\left(d_{\nu+1}\right)
$$

and the expected mean reward per step for $n-1$ transitions is

$$
\bar{V}_{n}(\pi) \equiv V_{n}(\pi) / n \text {. }
$$

In general, (3.1) does not converge as $n \rightarrow \infty$, and so refering to the minmax doctrine let us define the value of policy $\pi$ by

$$
\bar{V}(\pi) \equiv \lim _{n \rightarrow \infty} \inf \bar{V}_{n}(\pi),
$$

where the right hand side means the vector each component of which is the inferior limit of the corresponding component of $\bar{V}_{n}(\pi)$.

Similarly to the foregoing paragraph, we set the correspondence between the semi-order in $\mathfrak{v}(I)$ (space of $V(\pi)$ ) and that in $\Pi$ as follows,

$$
\begin{aligned}
& V\left(\pi_{1}\right) \leqq \bar{V}\left(\pi_{2}\right) \rightleftarrows \pi_{1} \leqq \pi_{2} \\
& \bar{V}\left(\pi_{1}\right)<\bar{V}\left(\pi_{2}\right) \rightleftarrows \pi_{1}<\pi_{2} \\
& \bar{V}\left(\pi_{1}\right)=\bar{V}\left(\pi_{2}\right) \rightleftarrows \pi_{1}=\pi_{2}
\end{aligned}
$$

and $\pi^{*}$ is an optimaI policy, if $\pi^{*} \geqq \pi$ for all $\pi \in \Pi$. We may point out here only that the lattice theoretical interpretation to the present case can be given quite similarly to the foregoing case of $\beta<1$. 
Now, for two policies

$$
\pi_{1}=\left(d_{1}^{1} d_{2}^{1} \cdots\right) \text { and } \pi_{2}=\left(d_{1}^{2} d_{2}^{2} \cdots\right)
$$

we set

$$
\begin{aligned}
V_{m, n}\left(\pi_{1}, \pi_{2}\right) & \equiv \sum_{\nu=0}^{m-1} P_{\nu}\left(\pi_{1}\right) r\left(d_{\nu+1}^{1}\right)+P_{m}\left(\pi_{1}\right) \sum_{\nu=0}^{n-1} P_{\nu}\left(\pi_{2}\right) r\left(d_{\nu+1}^{2}\right) \\
& =m \bar{V}_{m}\left(\pi_{1}\right)+n P_{m}\left(\pi_{1}\right) \bar{V}_{n}\left(\pi_{2}\right) \\
\bar{V}_{m, n}\left(\pi_{1}, \pi_{2}\right) & \equiv V_{m, n}\left(\pi_{1}, \pi_{2}\right) /(m+n) .
\end{aligned}
$$

Then we get the following

Lemma 3. 1. $\lim _{m \rightarrow \infty} \inf \bar{V}_{m, n}\left(\pi_{1}, \pi_{2}\right)=\bar{V}\left(\pi_{1}\right)$

$$
\lim _{n \rightarrow \infty} \inf \bar{V}_{m, n}\left(\pi_{1}, \pi_{2}\right)=P_{m}\left(\pi_{1}\right) \bar{V}\left(\pi_{2}\right) \equiv \bar{V}\left(d_{1}^{1} \cdots d_{m}^{1} \pi\right)
$$

Theorem 3.1. If there exists an integer $n_{1}$ such that

$$
\bar{V}_{1, n}(d, \pi) \geqq \bar{V}_{n+1}(\pi) \text { for all } n \geqq n_{1}
$$

then $d^{(\infty)} \geq \pi$.

Proof. We define a monotone operator $L(d)$ associated with each $d \in D$ by

$$
L(d) v=r(d)+P(d) v \quad(v \in \mathfrak{p})
$$

Then, from our assumption, we get

$$
L(d) V_{n}(\pi) \geqq V_{n+1}(\pi) \quad n \geqq n_{1}
$$

Consequently $\quad L^{m}(d) V_{n}(\pi) \geqq L^{m-1}(d) V_{n+1}(\pi) \geq \cdots \geq V_{n+m}(\pi)$.

That is

$$
\bar{V}_{m, n}(d, \pi) \geqq \bar{V}_{m+n}^{(\infty)}(\pi)
$$

Hence

$$
\lim _{m \rightarrow \infty} \inf \bar{V}_{m, n}\left(d^{(\infty)}, \pi\right) \geq \lim _{m \rightarrow \infty} \inf V_{m+n}(\pi)
$$

Therefore

$$
\begin{gathered}
\bar{V}\left(d^{(\infty)}\right) \geqq V(\pi) \\
d_{(\infty)} \geqq \pi .
\end{gathered}
$$

Thus we get

Theorem 3.2. Assume that $\lim _{n \rightarrow \infty} \bar{V}_{n}(\pi)=\bar{V}(\pi)$ exists for a policy $\pi$. If $(d \pi)>\pi$, then $d^{(\infty)}>\pi$, and if $(d \pi)<\pi$, then $d^{(\infty)}<\pi$.

Proof. By the assumption there exist two vectors $\alpha, \beta$ such that

$$
L(d) V_{n}(\pi) \geqq(n+1) \alpha>(n+1) \beta \geqq V_{n+1}(\pi) \text { for } n \text { large. }
$$

Since the operator $L(d)$ is monotone,

$$
\begin{aligned}
L^{2}(d) V_{n}(\pi) & \geqq L(d)(n+1) \alpha \geqq L(d)(n+1) \beta \geqq L(d) V_{n+1}(\pi) \\
& \geqq(n+2) \alpha>(n+2) \beta \geqq V_{n+2}(\pi)
\end{aligned}
$$


By repeating the operation, we get

$$
L^{m}(d) V_{n}(\pi) \geqq(n+m) \alpha>(n+m) \beta \geqq V_{n+m}(\pi) .
$$

Deviding each term by $(n+m)$ and letting $m \rightarrow \infty$, we have

$$
\bar{V}\left(d^{(\infty)}\right) \geqq \alpha>\beta \geq V(\pi)
$$

Hence

$$
d^{(\infty)}>\pi \text {. }
$$

The second part of the theorem is proved in the same way.

Now, the following fact is well known in the theory of Markov chain.

Lemma 3.2. For any $d \in D$

$$
\lim _{n \rightarrow \infty}\left(1+P(d)+P(d)^{2}+\cdots+P(d)^{n}\right) /(n+1)=P_{d}
$$

exists and $P_{d} P(d)=P(d) P_{a}=P_{d}^{2}=P_{a}$.

From this lemma we get

Theorem 3.3. If $\pi=d^{(\infty)}$, then $\left(d^{(n)} \pi\right)=\pi$ for $n=1,2, \cdots$.

Proof. By assumption $\bar{V}(\pi)=\bar{V}\left(d^{(\infty)}\right)$. On the other hand, by lemma 3.2, $\bar{V}\left(d^{(\infty)}\right)=P_{d} r(d)$ and $P(d)^{n} \bar{V}\left(d^{(\infty)}\right)=P_{d} r(d)=\bar{V}\left(d^{(\infty)}\right)$. Therefore, $P(d)^{n} \bar{V}(\pi)=P(d)^{n} \bar{V}\left(d^{(\infty)}\right)=\bar{V}\left(d^{(\infty)}\right)=\bar{V}(\pi)$. Consequently, by lemma 3.1, we get $\left(d^{(n)} \pi\right)=\pi$.

Finally we observe

Theorem 3.4. If $\pi \equiv\left(d_{1} d_{2} \cdots\right)$ is optimal, then

i) $\pi=\left(d_{1}^{(n)} \pi\right) \quad n=1,2, \cdots$

ii) $\pi$ and $d_{1}^{(\infty)}$ belong to the same class $\Pi^{*} \equiv\left\{\pi ; P\left(d_{1}\right) \bar{V}(\pi)=\bar{V}(\pi)\right\}$.

Proof. First, we see that lemma 2.2 holds in case of $\beta=1$ too; for any two policies $\pi_{1}$ and $\pi_{2}$

$$
\text { if } \pi_{1} \leqq \pi_{2} \text {, then }\left(d \pi_{1}\right) \leqq\left(d \pi_{2}\right) \text { for all } d \in D \text {. }
$$

In fact, since $P(d)$ is also a monotone operator, from $\bar{V}\left(\pi_{1}\right) \leqq \bar{V}\left(\pi_{2}\right)$ we get $P(d) V\left(\pi_{1}\right) \leqq P(d) V\left(\pi_{2}\right)$, that is $\bar{V}\left(d \pi_{1}\right) \leqq \bar{V}\left(d \pi_{2}\right)$ which leads to (3.2).

Now, because of optimality of $\pi$, we have $\left(d_{1} d_{2} \cdots\right) \geq\left(d_{2} d_{3} \cdots\right)$ and by (3. 2) $\left(d_{1} d_{1} d_{2} \cdots\right) \geqq\left(d_{1} d_{2} \cdots\right)$ or $\left(d_{1} \pi\right) \geqq \pi$, while $\left(d_{1} \pi\right) \leqq \pi$. Hence $\left(d_{1} \pi\right)=\pi$, that is $P\left(d_{1}\right) V(\pi)=\bar{V}(\pi)$. Operating $P\left(d_{1}\right) n-1$ times on both sides, we get $P\left(d_{1}\right)^{n} \bar{V}(\pi)=\bar{V}(\pi)$ which proves (i). On the other hand, from $\bar{V}\left(d_{1}^{(\infty)}\right)$ $=P_{d} r\left(d_{1}\right)$ we get $P\left(d_{1}\right) \bar{V}\left(d_{1}^{(\infty)}\right)=P_{d_{1}} r\left(d_{1}\right)=\bar{V}\left(d_{1}^{(\infty)}\right)$.

Thus, together with the equation $P\left(d_{1}\right) \bar{V}(\pi)=\bar{V}(\pi)$ obtained above, part (ii) of the theorem was proved.

Corollary. If $\pi \equiv\left(d_{1} d_{2} \cdots\right)$ is optimal,

$$
\max _{d \in D} P(d) \bar{V}(\pi)=P\left(d_{1}\right) \bar{V}(\pi)=\bar{V}(\pi) .
$$


Even if $\pi \equiv\left(d_{1} d_{2} \cdots\right)$ is optimal, the equivalence $\pi=d_{1}^{(\infty)}$ may not be concluded (except the special case of $r\left(d_{1}\right)=V(\pi)$ ) which was necessary consequence in case of $\beta<1$. However we may think of that the policy $\mathrm{d}_{1}^{(\infty)}$ is 'nearly optimal' in the sense of theorem 3.4 and corollary to it.

By the way, in relation to our equation $P\left(d_{1}\right) V(\pi)=V(\pi)$, there is Bellman's theorem ([1], p. 329). However, since $\left(a_{i j}(\mathrm{q})\right)$ in his theorem is a Markov matrix as well as our $P\left(d_{1}\right)$, the solution has the form $\alpha y_{i}+\beta$ $(i=1,2, \cdots, N)$ where $\alpha$ and $\beta$ are arbitrary real constants.

\section{§4. A general formulation.}

We may generalize the state space to $k$-dimensional vector space $R^{k}$ and the atcion space to $m$-dimensional vector space $R^{m}$; decision function $d(s)\left(s \in R^{k}\right)$ is a mapping from $R^{k}$ into $R^{m}$, and $D$ is the set of all decision functions, while immediate reward $r\left(s_{1}, s_{2}\right)$ corresponding to a transition of state $s_{1} \rightarrow s_{2}$ is a real valued random variable.

Suppose that states $s=s(t)$ are observed at equally spaced discrete time points $t=0,1,2, \cdots$ and an action $d(s)$ is decided each time according to the state and let the transition probability distribution function associated with the decision function $d$ be

$$
\begin{gathered}
F\left(s_{0}, s ; d\right)=\operatorname{Pr}\left(s(n) \leqq s \mid s(n-1)=s_{0} ; d\left(s_{0}\right)\right) \\
(n=1,2, \cdots)
\end{gathered}
$$

that is independent of time $n$, where $s_{0}$ and $s$ are $k$-dimensional vectors and $\leqq$ means the semi-order defined similarly to that in $\S 2$.

Policy is a sequence of decision functions; $\pi \equiv\left(d_{1} d_{2} \cdots\right)$, a point of policy space $\Pi$. The $n$ step transition probability distribution function for a policy $\pi \equiv\left(d_{0} d_{1} d_{2} \cdots\right)$ is given by

$$
\begin{gathered}
F_{n}\left(s_{0}, s ; \pi\right)=\int d F\left(s_{0}, s_{1} ; d_{0}\right) d F\left(s_{1}, s_{2} ; d_{1}\right) \cdots d F\left(s_{n-2}, s_{n-1} ; d_{n-2}\right) F\left(s_{n-1}, s ; d_{n-1}\right) \\
n=1,2, \cdots
\end{gathered}
$$

where $F_{1}\left(s_{0}, s ; \pi\right)=F\left(s_{0}, s ; d_{0}\right)$.

The expected immediate reward started with state $s_{1}$ and decision $d\left(s_{1}\right)$ is

$$
r\left(s_{1}, d\left(s_{1}\right)\right)=\int r\left(s_{1}, s_{2}\right) d F\left(s_{1}, s_{2} ; d\right)
$$

and the expected total reward for a policy $\pi \equiv\left(d_{0} d_{1} d_{2} \cdots\right)$ with initial state $s_{0}$ is given by

$$
V\left(\pi ; s_{0}\right)=\sum_{n=0}^{\infty} \beta^{n} \int r\left(s ; d_{n}(s)\right) d F_{n}\left(s_{0}, s ; \pi\right)
$$

where $\beta$ is a discount factor and $0 \leqq \beta<1$.

Now, into the space $\mathfrak{v}$ of real valued functions of $k$ real variables we can introduce semi-order and norm in a quite similar way as before. If 
the subspace $\mathfrak{v}(I)=\{V(\pi) ; \pi \in I I\}$ of $\mathfrak{v}$ is a complete vector lattice and if

$$
\sup _{\pi \in \pi} V(\pi)=V\left(\pi^{*}\right), \quad \pi^{*} \in I I
$$

then $\pi^{*}$ is an optimal policy, and the theorems $2.1 \sim 2.3$ hold in the same form.

In case of $\beta=1$, we may consider

$$
\begin{aligned}
& \bar{V}_{n}\left(\pi, s_{0}\right)=(n+1)^{-1} \sum_{\nu=0}^{\infty} \int r\left(s, d_{\nu}(s)\right) d F_{\nu}\left(s_{0}, s ; \pi\right) \\
& \bar{V}\left(\pi, s_{0}\right)=\lim _{n \rightarrow \infty} \inf \bar{V}_{n}\left(\pi ; s_{0}\right),\left(s_{0} \in R^{k}\right)
\end{aligned}
$$

and we can proceed in almost same way as preceding paragraph. The dynamic form of maximum expected reward $V_{n}\left(\pi, s_{0}\right)$ for an optimal policy $\pi \equiv\left(d_{1} d_{2} \cdots\right)$ in $n$ steps started in state $s_{0}$ may be given by

$$
V_{n}\left(\pi ; s_{0}\right)=\max _{d_{1} \in D}\left[r\left(s_{0}, d_{1}\left(s_{0}\right)\right)+\beta \int V_{n-1}(T \pi ; s) d F\left(s_{0}, s ; d_{1}\left(s_{0}\right)\right)\right], 0 \leqq \beta \leqq 1 .
$$

Acknowledgement. This note is one of the studies developed in the DP Developing Group sponsored by the Japanese Union of Scientists and Engineers. The author would like to express his thanks to Prof. T. Kitagawa, chief of the group, and the other members for the discussions given by them.

\section{Tokyo Woman's Christian College}

\section{References}

[1] R. Bellman: Dynamic Programming, Princeton (1957).

[2] R.. A. Howard: Dynamic Programming and Markov Processes, MIT and Wiley (1960).

[3] D. Blackwell: Discrete Dynamic Programming, Ann. Math. Statist., Vol. 33 (1962), 719-726. 